\title{
Laparoscopic splenic-preserving distal pancreatectomy for trauma in a child
}

\author{
Marcus M. Malek • Sohail R. Shah • \\ Timothy D. Kane
}

Published online: 23 February 2010

(C) Springer Science+Business Media, LLC 2010

\begin{abstract}
Purpose To reduce the risks associated with splenectomy, there has been a trend toward splenic preservation when performing distal pancreatectomy. Although laparoscopy has gained almost universal acceptance, it is still not utilized often for pancreatic surgery in children. In fact, review of the literature shows only a few case series of splenic-preserving laparoscopic distal pancreatectomy, and even less reported experience in the pediatric population [1-3]. To our knowledge, there has been only one other report of laparoscopic spleen-preserving distal pancreatectomy in the setting of trauma in a child [4].

Methods A 13-year-old boy suffered a handlebar injury to the abdomen secondary to a dirt bike accident. He did not report loss of consciousness but complained of abdominal pain. CT scan revealed a grade II splenic laceration with a transected distal pancreas. He was hemodynamically stable with a large contusion in the left upper quadrant. Laboratory evaluation showed that his hematocrit was stable but was significant for elevated amylase and lipase. During his hospitalization, he developed significant abdominal distension and his amylase and lipase continued to rise. $\mathrm{He}$
\end{abstract}

Electronic supplementary material The online version of this article (doi:10.1007/s00464-010-0961-x) contains supplementary material, which is available to authorized users.

M. M. Malek · S. R. Shah · T. D. Kane $(\bowtie)$

Division of Pediatric General and Thoracic Surgery, Children's Hospital of Pittsburgh of UPMC, University of Pittsburgh

Medical Center, 4401 Penn Ave., Floor 4, Pittsburgh, PA 15224, USA

e-mail: kanetd@upmc.edu

M. M. Malek

e-mail: marcus.malek@chp.edu was taken to the operating room for definitive management of his pancreatic injury 72 hours after his initial injury. Results In the operating room, a central line was placed so that he could receive total parenteral nutrition postoperatively. The video demonstrates the performance of a laparoscopic spleen-preserving distal pancreatectomy. There was a fair amount of old blood seen in the left upper quadrant and behind the pancreas. There was no evidence of active bleeding. The operation was performed without complication and with preservation of the splenic vessels. Conclusions The postoperative course was very smooth with the child resuming diet by postoperative day (POD) 4 . He was sent home on POD 7. This video demonstrates that in the proper trauma patient, a minimally invasive approach to a spleen-preserving distal pancreatectomy can still be performed.

Keywords Pediatric laparoscopy $\cdot$ Pancreas · Trauma

Disclosures Drs. Marcus M. Malek, Sohail R. Shah, and Timothy D. Kane have no conflicts of interest or financial ties to disclose.

\section{References}

1. Sasaki A, Nitta H, Nakajima J, Obuchi T, Baba S, Wakabayashi G (2008) Laparoscopic spleen-preserving distal pancreatectomy with conservation of the splenic artery and vein: report of three cases. Surg Today 38:955-958

2. Bruzoni M, Sasson AR (2008) Open and laparoscopic spleenpreserving, splenic vessel-preserving distal pancreatectomy: indications and outcomes. J Gastrointest Surg 12:1202-1206

3. Melotti G, Cavallini A, Butturini G, Piccoli M, Delvecchio A, Salvi C, Pederzoli (2007) Laparoscopic distal pancreatectomy in children: case report and review of the literature. Ann Surg Oncol 14:1065-1069

4. Reynolds EM, Curnow AJ (2003) Laparoscopic distal pancreatectomy for traumatic pancreatic transection. J Pediatr Surg 38:E7-E9 